\section{LA ESTRUCTURA METALICA DEL CASINO «GRAN MADRID», TORRELODONES/MADRID/ESPAÑA}

\author{
Jesús Ortiz Herrera, \\ Juan Antonio Domínguez Montero, \\ María Jesús Izquierdo Martínez, \\ Ingenieros de Caminos.
}

\author{
José Espinosa González, \\ Aparejador $(*)$
}

$841-25$

\section{Sinopsis}

Se describe en este artículo la solución estructural metálica adoptada en los niveles superiores del edificio del Casino «Gran Madrid».

Las piezas principales de cubierta se han resuelto en forma de vigas trianguladas de canto elevado, ligeramente variable, con un sistema de triangulación del tipo Warren simple. Los apoyos de dichas vigas son de neopreno zunchado.

\section{La disposición Pratt ha sido la comúnmente escogida para las demás vigas.}

El resto de los dinteles, así como los soportes, están formados por perfiles laminados en sus distintas variantes.

\section{Introducción}

Esta obra - promovida por la Sociedad "Casino de Juego Gran Madrid» - ha sido realizada en un plazo muy reducido, para la envergadura y complejidad de la construcción, habiendo jugado precisamente un importante papel a efectos de la celeridad de la misma lque era uno de los condicionantes básicos del proyecto), la adopción de una solución metálica para toda la zona superior del edificio, en la cual se incluyen las máximas luces y solicitaciones.

\footnotetext{
(*) Miembros del gabinete "HCA - Proyectistas», Madrid.
}

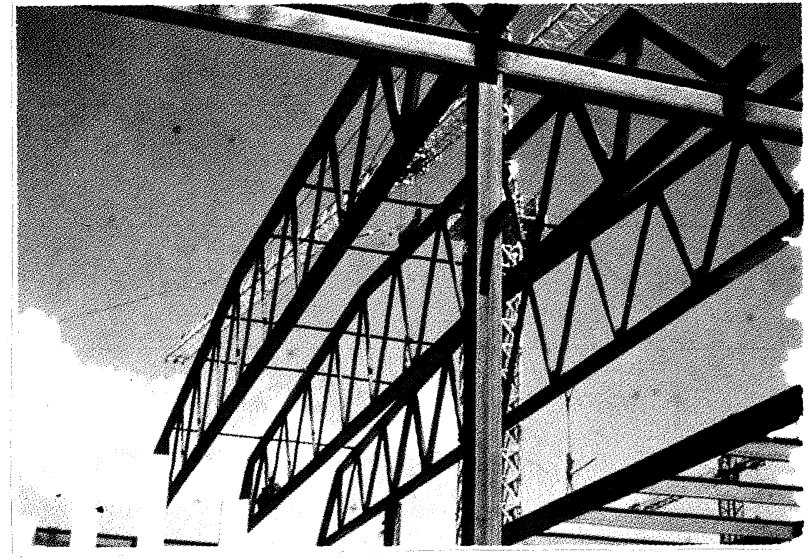

Vigas trianguladas principales durante el montaje.

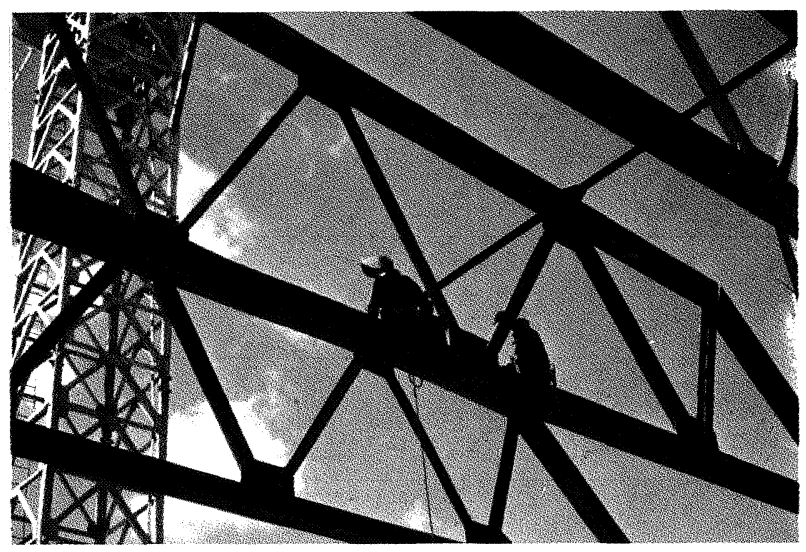

Detalle de las mismas vigas.

La citada necesidad imperativa de un plazo de ejecución breve y la complejidad de los condicionantes estructurales hacían particularmente indicada la solución metálica, arrancando a nivel de salas de juego, por aparecer elementos con cargas muy importantes, luces notables, soportes en número reducido y dispersos por condiciones arquitectónicas, forjado intermedio (entreplanta) suspendido de las piezas de cubierta, y otras condiciones particulares posteriormente descritas. En efecto, las ventajas de la construcción metálica en lo relativo a rapidez de ejecución son bien conocidas, así como su versatilidad y capacidad para resolver configuraciones estructurales difíciles $\mathrm{y} / \mathrm{o}$ con grandes solicitaciones. El gabinete HCA-Proyectistas fue el encargado de realizar el análisis y diseño estructural de la parte metálica del Casino.

\section{Descripción de la solución estructural}

Interesa destacar, en primer lugar, que las cargas verticales de cubierta son de orden de magnitud 
elevado (típicamente de 730 a $865 \mathrm{kp} / \mathrm{m}^{2}$, y de hasta $1.150 \mathrm{kp} / \mathrm{m}^{2}$ en algunas zonas), debido a la adopción de placas de hormigón prefabricadas como sistema de cobertura y a la existencia de un total importante de cargas muertas (impermeabilización, placas rígidas de hormigón filtrante, maquinaria en algunas zonas, falso techo, superestructura de decoración e iluminación), aparte de la incidencia de peso propio de la estructura metálica y la sobrecarga de uso mínima o acciones ecológicas. Estos valores de cargas totales, muy superiores a los de una cubierta ligera ordinaria, alteran por sí solos, e independientemente del resto de factores luego detallados, los conceptos usualmente válidos para el tratamiento estructural de cubiertas convencionales. La disposición de un forjado de entreplanta, también con carga total importante $\left(750 \mathrm{kp} / \mathrm{m}^{2}\right)$, cuyo perímetro interior no podría sustentarse en soportes inexistentes en las grandes salas diáfanas, y ciertas escaleras, que debían también plantearse como elementos colgados de las grandes piezas de cubierta, abundaban en la producción de solicitaciones muy considerables en dichas piezas. Incluso alguna de las mismas recibe, además, embrochaladas contra su cordón inferior, jácenas transversales de porte considerable, lo cual no sólo repercute en los valores de las solicitaciones, sino que también crea fuertes complicaciones constructivas por tener que resolverse en un mismo nudo, frecuentemente, el encuentro de barras principales

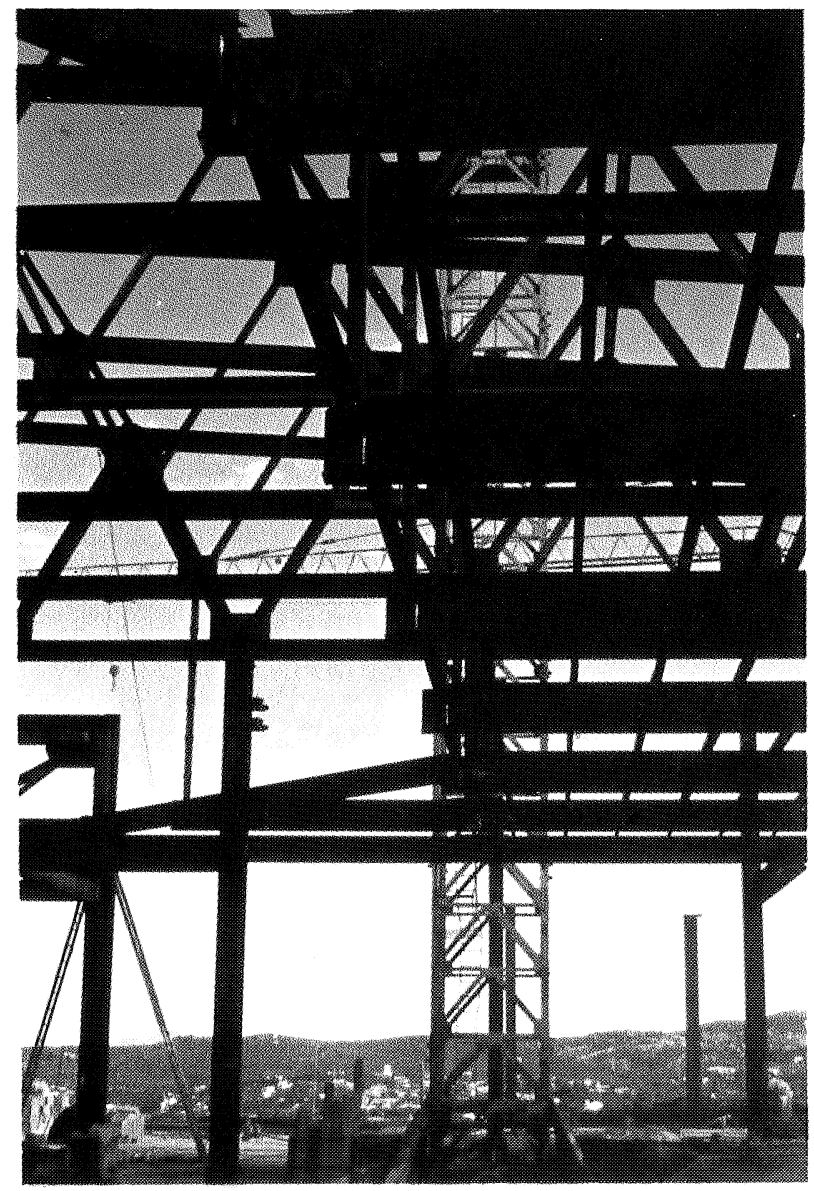

Extremo del forjado de entreplanta, suspendido de cubierta.

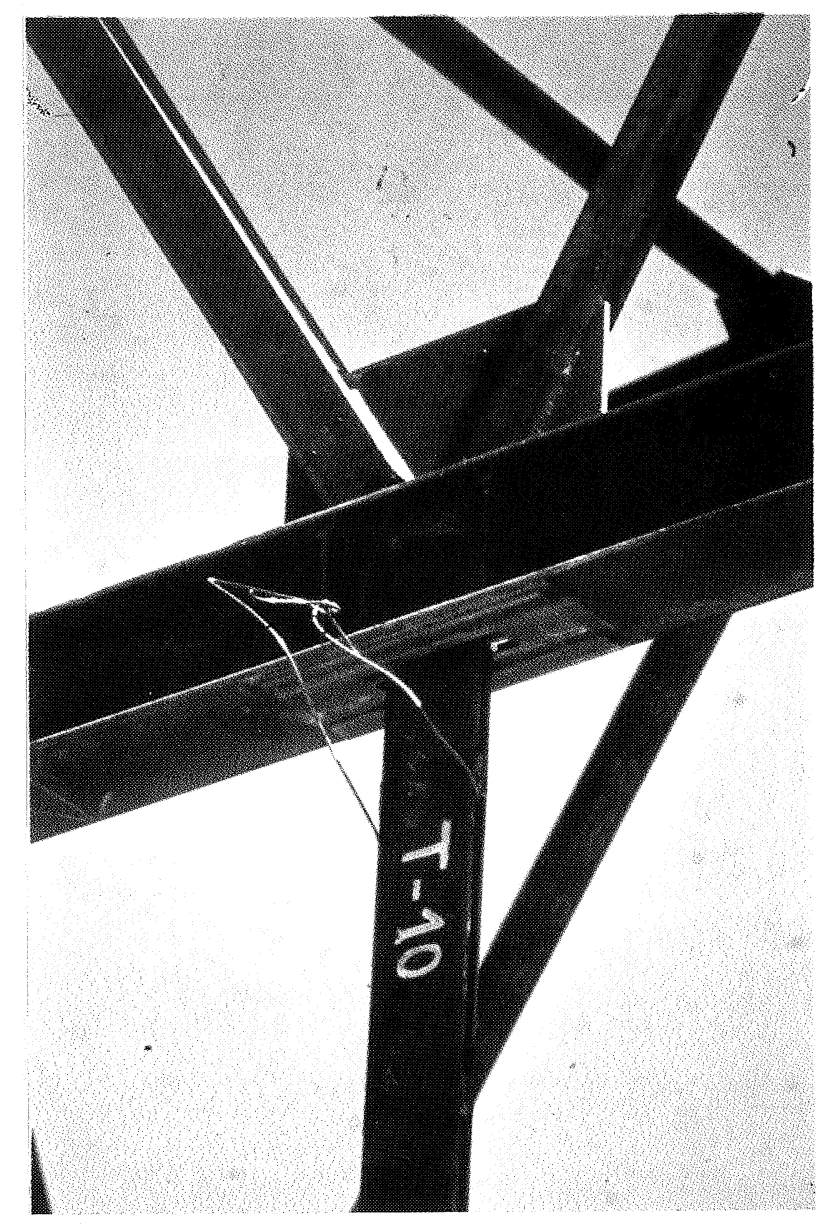

Detalle de tirante (extremo superior).

de gran sección, junto con un tirante sustentante del forjado de entreplanta, y jácenas transversales de canto importante; éstas y otras dificultades del diseño se tuvieron muy en cuenta desde el principio del proceso, ya que no sólo condicionaban fuertemente los tipos de soluciones posibles, sino que se revelaron desde el primer momento como un aspecto del proyecto que requería la máxima dedicación, por encima del mero análisis.

Las piezas principales de cubierta, de 41 y 37 metros, en diversas variantes, no pueden considerarse de luces extraordinarias (sobre todo tratándose de una solución metálica), pero debido al valor considerable de las acciones correspondientes experimentan solicitaciones no usuales en piezas de cubierta, con un momento global pésimo del orden de los $1.000 \mathrm{mt}$ (valor característico). Las reacciones, también de valor considerable (alrededor de $90 \mathrm{t}$; hasta $232 \mathrm{t}$ en el apoyo intermedio de una pieza hiperestática), planteaban problemas para su transmisión a los soportes, cuya separación impuesta era de dos o tres veces el módulo establecido como intereje de las piezas principales. El canto de éstas era libre dentro de algunos márgenes, respetando ciertos perfiles generales, valores de pendientes, etc.

Con objeto de hacer frente a estos condicionantes, las piezas principales de cubierta se han resuelto en 


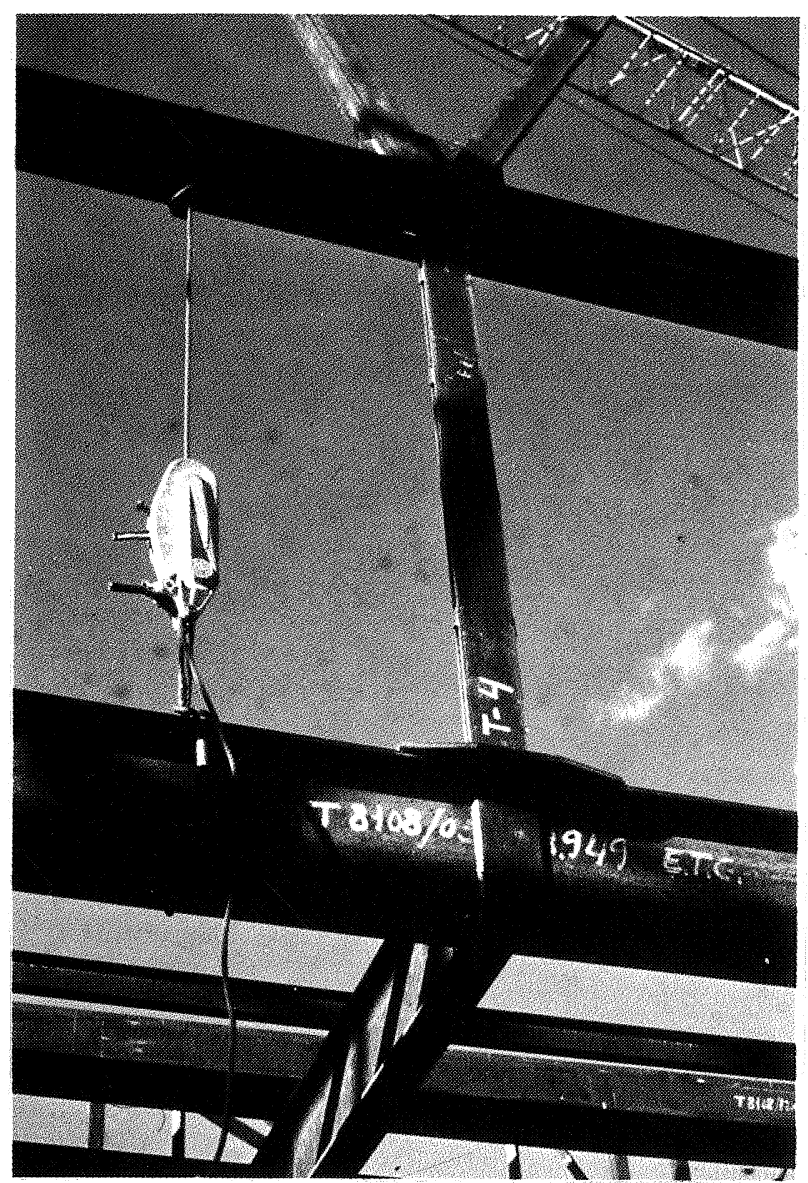

Detalle de tirante (extremo inferior).

forma de vigas trianguladas de canto elevado, ligeramente variable, con valor máximo central de $4 \mathrm{~m}$ (alrededor del décimo de la luz), valor que resultaría desproporcionado para otro tipo de cubiertas más ligeras, pero que en este caso permite satisfacer un doble objetivo: por un lado, resolver la totalidad de los elementos con perfilería (secciones máximas a base de perfiles HEB reforzados con chapas), evitando pues la utilización de secciones armadas; $y$, por otro lado, acotar adecuadamente la flexibilidad de dichas piezas de cubierta, que convenía fueran poco deformables debido a la cantidad de elementos estructurales y no estructurales suspendidos de las mismas, sin impedir por ello la plena utilización resistente de las secciones de las barras (además, no obstante, se dispuso un conjunto de contraflecha de ejecución adecuada en todas las piezas principales).

Tras diversos tanteos, se adoptó la tipología Warren "simple» como sistema de triangulación de las piezas principales, ya que la modulación del edificio permitía encajar todos los puntos de aplicación de cargas localizadas (tirantes, vigas transversales) manteniendo una triangulación regular del tipo indicado, sin recurrir a otras más prolijas, de mayor número de nudos y elementos; de esta forma se conseguía una triangulación «limpia» y simple, conveniente a efectos de aminoración de esfuerzos secundarios y de simplicación de la ejecución en taller (gran número de nudos en el conjunto de la obra). La única des- ventaja de la tipología Warren en este caso la constituía un relativo incremento de la longitud individual de los elementos comprimidos, con aumento a su vez de las flexiones parásitas inducidas en los mismos por la aplicación de cargas repartidas; pero la incidencia de estos aspectos se valoró numéricamente como poco importante, no justificando en absoluto la adopción de otras tipologías posibles (Warren «subdividida», Pratt, etc.), las cuales habrian anulado o reducido las ventajas anteriormente mencionadas.

La disposición Pratt y otras se han adoptado por otras vigas trianguladas existentes, de luces menores que las anteriormente indicadas. El aspecto más singular es la existencia de cerchas «embrochaladas» (vigas trianguladas apoyadas o pasando continuas a través de otras transversales), circunstancia motivada por la dispersión de soportes en algunas zonas. En total se tienen veintisiete tipos diferentes de vigas trianguladas en toda la estructura.

El resto de los dinteles, allí donde las luces y cargas lo permiten, están constituidos por perfiles laminados en distintas variantes, tales como IPN simples o reforzados, perfiles HEB (en determinadas piezas de canto restringido) y perfiles «Boyd», con canto de hasta $80 \mathrm{~cm}$ (vigas de $18 \mathrm{~m}$ de luz en la marquesina de la entrada). Mención aparte merecen los dinteles de borde sobre los que apoyan las grandes vigas

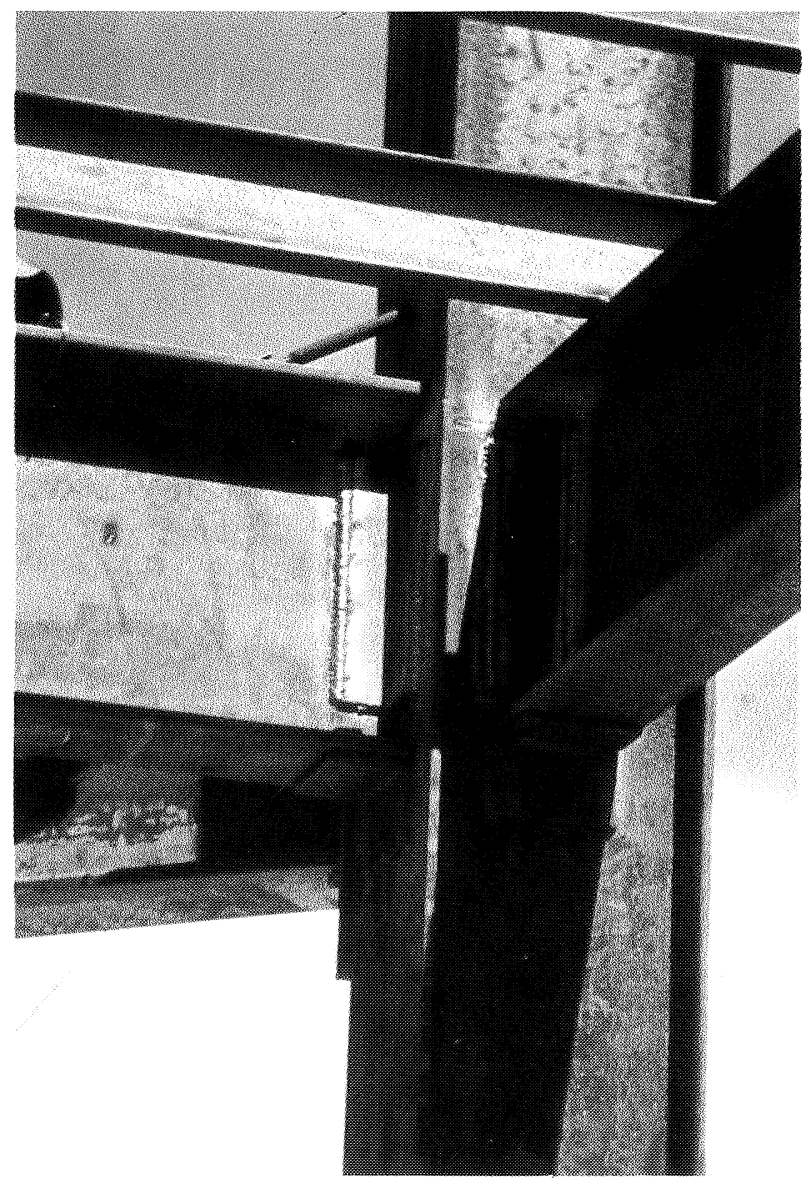

Apoyo simple de dinteles secundarios. 


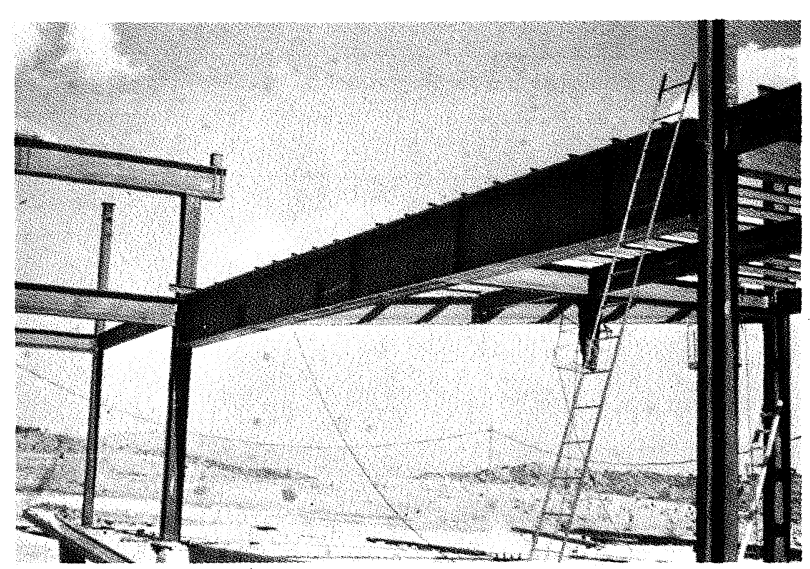

Viga armada.

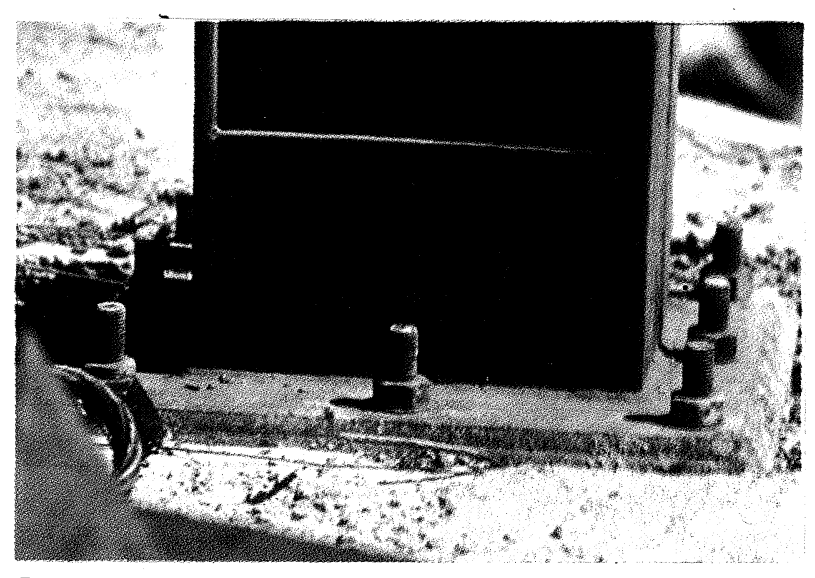

Basa de soporte.

trianguladas de cubierta; dichos dinteles reciben las importantes reacciones transmitidas por las citadas piezas principales, pero al ser de luces moderadas $(7,5$ y $11,25 \mathrm{~m})$ y de continuidades favorables, pudieron resolverse a base de perfiles IPN apareados, con grandes refuerzos en algunas zonas; a pesar de ello se tuvieron problemas de canto, el cual estaba fuertemente limitado en algunos puntos de dichos dinteles. En el conjunto de la estructura, solamente en un caso se acudió a una viga armada, para resolver un vano isostático de $15 \mathrm{~m}$ de luz, con una doble T soldada de $95 \mathrm{~cm}$ de canto.

Una buena parte de los dinteles menores se ha planteado con carácter isostático, a efectos de clarificar el comportamiento estructural en zonas de distribución irregular $\mathrm{y} / \mathrm{o}$ con apoyos deformables (tirantes, brochales). No obstante, convenía resolver alguna de las piezas en tales condiciones como vigas continuas; para salvar la complicación indudable que suponía la introdución en el cálculo de los descensos diferenciales de los apoyos, el análisis del estado límite de agotamiento por flexión de dichas piezas se efectuó por métodos plásticos, en los cuales los descensos diferenciales antedichos (y otras acciones productoras de sistemas de esfuerzos autoequilibrados) no afectan a los valores de las cargas últimas; las condiciones de aplicabilidad del cálculo plástico se verificaron escrupulosamente en dichos casos.
Otros dinteles especiales (por ejemplo, los ya mencionados de apoyo de las cerchas principales), se han dimensionado observando criterios estrictamente elásticos.

El pandeo lateral de los dinteles está impedido, bien por el enlace con el forjado, bien por piezas transversales unidas al forjado o a los soportes, que subdividen la luz en intervalos suficientemente reducidos. En algunos casos, para vigas paralelas a la orientación del forjado, se prevé un detalle especifico de conexión a este último, con misión exclusivamente arriostrante. Los dinteles que reciben las reacciones de las grandes piezas de cubierta, al estar constituidos por secciones cerradas (IPN apareados), de suficiente rigidez torsional, alcanzan valores suficientemente elevados del momento crítico de pandeo lateral, a pesar de su carácter exento.

Los nudos de los cordones superiores de las vigas trianguladas están inmovilizados transversalmente por un arriostrado dispuesto en el plano de cubierta, al cual se enlazan dichos nudos mediante un sistema de correas de atado. Dada la gran masa de la cubierta y estructuras suspendidas, y dada la relativa flexibilidad de los soportes, cuya intraslacionalidad transversal no fue posible efectuarla de un modo total (por condiciones arquitectónicas), se juzgó conveniente mejorar la estabilidad de la cubierta, con un mínimo incremento de coste, haciéndola internamente completa a base de introducir en un módulo un cajón de celosía (arriostrado doble superior e inferior, con diafragmas de celosía a cuartos de la luz). En el caso de vigas trianguladas menores no se han considerado precisas tales precauciones, dotándolas de arriostrados convencionales. Algunas de las vigas trianguladas, por ser continuas o prolongarse en voladizos, requieren el arriostramiento de ciertos nudos del cordón inferior, lo cual se lleva a cabo mediante tornapuntas inclinadas.

A efectos de limitar la incidencia de acciones térmicas se han dispuesto juntas de dilatación, según práctica habitual en estructuras de edificación; en algún caso la separación de las mismas se aproxima a los $50 \mathrm{~m}$, lo cual se estima aceptable en este caso dada la relativa flexibilidad (perpendicularmente a la junta) de los soportes metálicos, de gran altura. A efectos de formalizar los apoyos de las grandes vigas trianguladas con un comportamiento aproximado al teórico previsto en el análisis, se han utilizado apoyos de neopreno zunchado, de utilización poco común en la edificación, cuyo empleo venía recomendado en este caso por los valores importantes de los giros y desplazamientos horizontales que debían tomar dichos apoyos, entre otras razones.

Los soportes, según sus solicitaciones y alturas respectivas y las condiciones de arriostramiento en cada caso, se han resuelto con diversos esquemas: perfiles UPN apareados (en tubo), con o sin refuerzos; dos perfiles IPN en cruz; dos perfiles IPN en cajón (unidos por las alas), con o sin platabandas de re- 
Fig. 1. - Esqu má ue la estructura de cubierta.
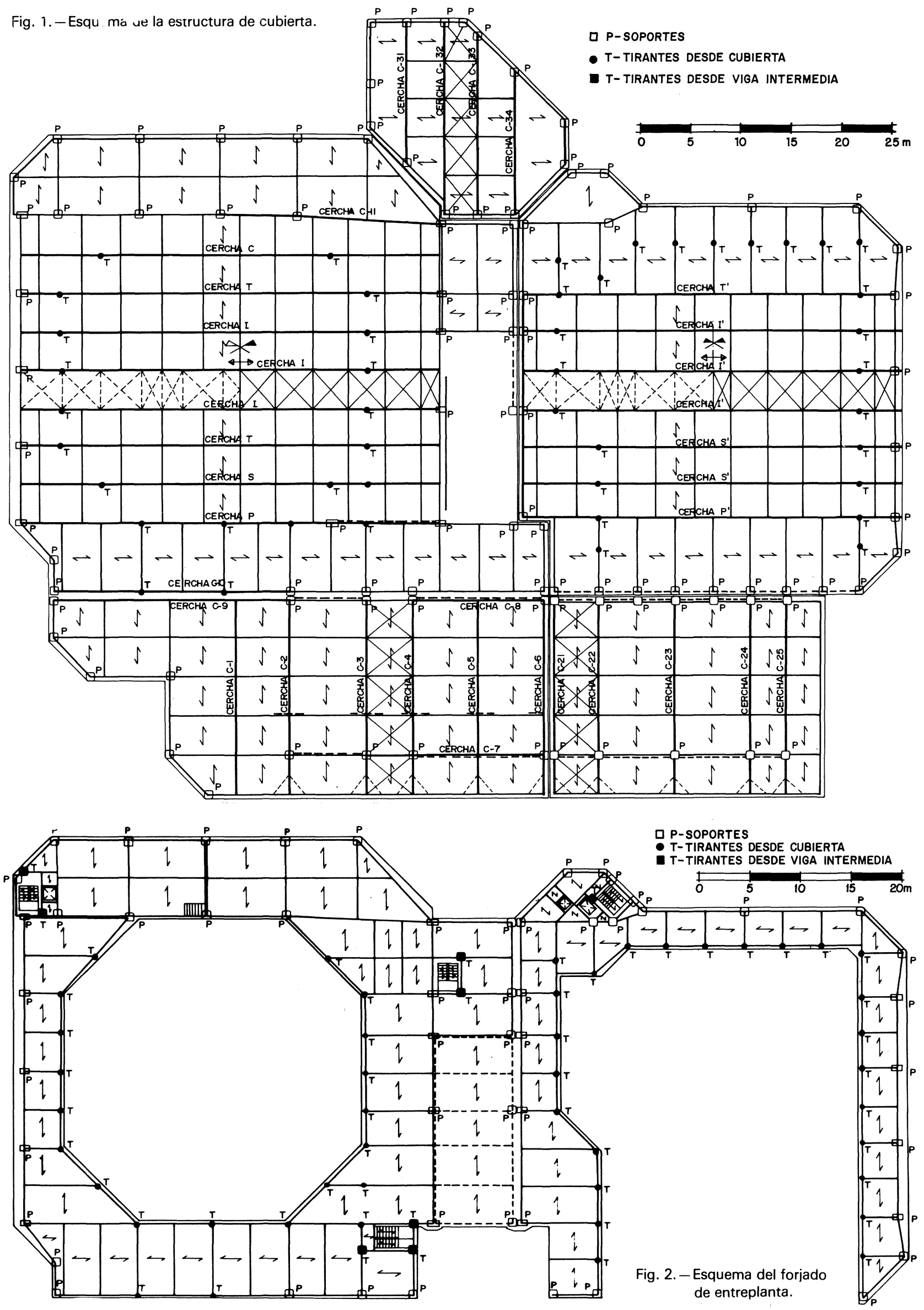
fuerzo. Un soporte especial, en cuyo interior debía discurrir el desagüe de la marquesina, quedando practicable, se ha resuelto mediante cuatro perfiles UPN empresillados.

El tipo de acero empleado, dadas las condiciones favorables relativas al carácter de las solicitaciones, espesores de chapas, condiciones térmicas, etc., ha sido de la clase $\mathrm{A} 42 \mathrm{~b}$; solamente en un caso, para una variante de basas con espesores elevados (donde las condiciones arquitectónicas impedían las soluciones acarteladas con menores espesores), se juzgó recomendable una calidad más exigente, la A42d.

La protección ignífuga utilizada asegura una resistencia al fuego de 180 minutos, acorde con las reglamentaciones locales vigentes a este efecto, las cuales resultan manifiestamente conservadoras a juicio de los autores de este artículo.

En las figuras 1,2 y 3 se definen esquemáticamente las plantas de la estructura metálica y el alzado de algunas de las vigas trianguladas de la obra.
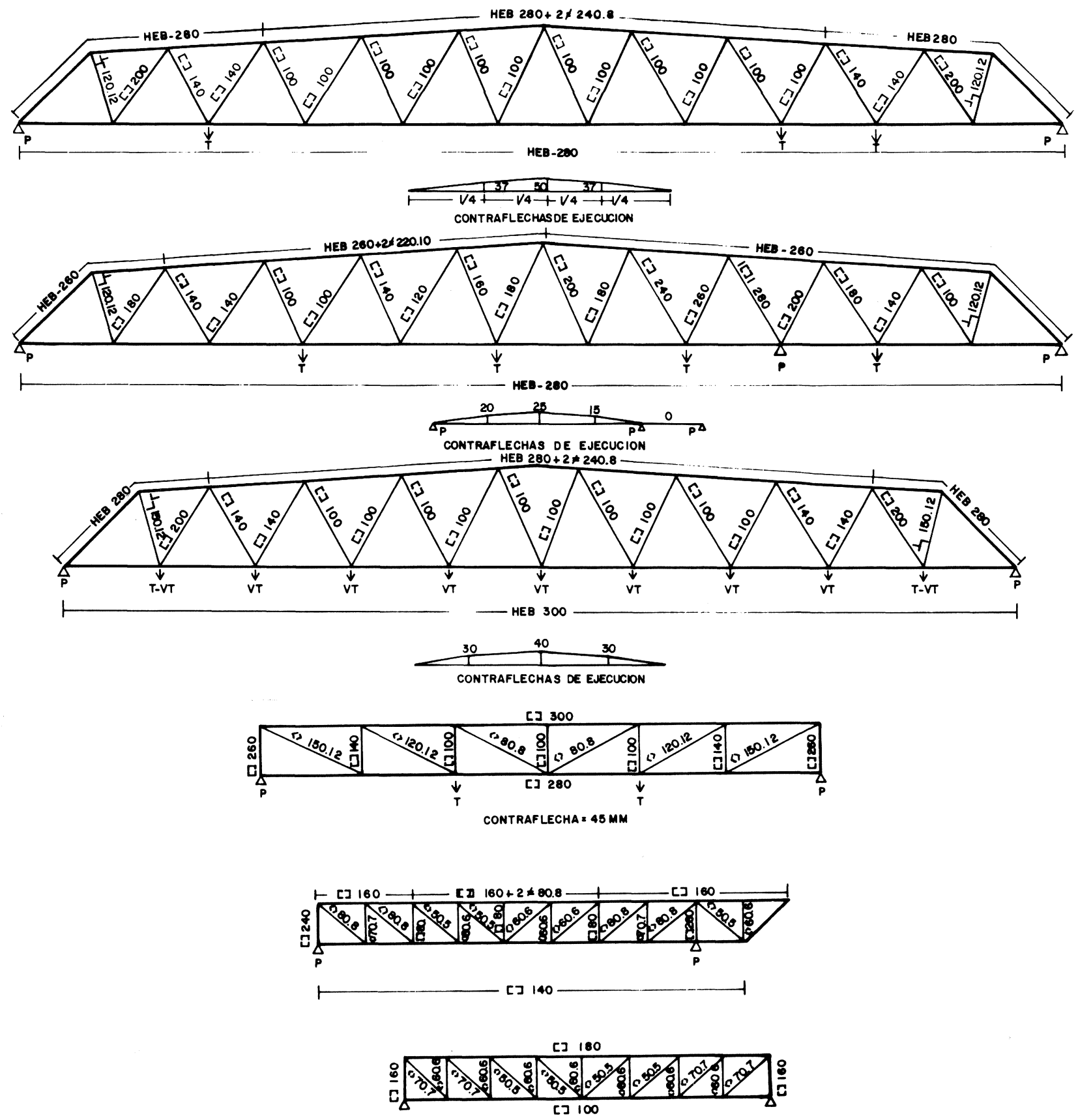

Fig. 3. - Algunos de los veintisiete tipos de vigas trianguladas.

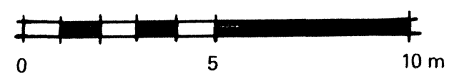




\section{Detalles constructivos}

La definición geométrica de los distintos detalles constructivos fue particularmente laboriosa, por incluir un número muy elevado de encuentros de piezas, pero se resolvió con una cantidad razonable de documentación gráfica a base de agrupar en familias los diferentes tipos de detalles análogos, complementándolos con tabulaciones de las combinaciones de valores posibles, tales como dimensiones de soldaduras, cartelas, casquillos, etc. No se describen aquí los detalles más convencionales, tales como encuentros de vigas con soportes, brochales, transiciones de perfiles, arriostramientos, basas, etc. En las figuras 4 y 5 pueden verse algunos de los nudos de las vigas trianguladas principales, en las que se ha procurado un diseño «limpio» y con cartelas de dimensiones relativamente moderadas para el porte de los perfiles involucrados. En la figura 6 se representa el apoyo de las grandes vigas trianguladas sobre el dintel de borde constituido por dos perfiles IPN (reforzados por platabandas en algunas zonas). La figura 7 representa la sección de la única viga armada de alma llena de la obra. Seguidamente se comentan los detalles más específicos de esta construcción, correspondientes a la recepción de los tirantes sobre las distintas piezas.

La unión de los tirantes a las piezas de cubierta y a las piezas de forjado colgado debía efectuarse necesariamente en obra $y$, por lo tanto, exigía un nudo accesible y soldaduras de ejecución no especialmente delicada. El necesario control de las posibles soldaduras comprometidas ejecutadas en obra, a pesar de garantizar la fiabilidad de las mismas en caso de resultado favorable, no resuelve el problema en caso de resultado negativo, ya que entonces el proyectista y el constructor se encuentran probablemente con una unión puesta parcialmente en carga, cuyo saneado y sustitución obligaría al apeo de los elementos sustentados (no estando garantizada por otra parte una mejor calidad de la soldadura reparada, en muchos casos); y la alternativa del refuerzo de la unión no siempre es posible o conveniente. Por ello se buscó una unión que involucrase cordones de soldadura exclusivamente de ángulo, con espesores de garantía moderados y longitudes algo sobredimensionadas; de esta forma se aminoraba la probabilidad de tener que recurrir a la reparación o al refuerzo de tales uniones de tirantes.

Por otra parte, salvo en algún caso, se evitó resolver tales encuentros de tirante mediante cartelas de nudo, en las dos variantes posibles, de cartela completa recibiendo barras formadas por perfiles apareados, o de cartela adosada inferiormente a un perfil simple. La primera de ambas variantes exige cartelas de grandes dimensiones, y es incompatible con barras de sección en $\mathrm{H}$ como las empleadas en los cordones de las vigas trianguladas más solicitadas; sin embargo esta solución se adoptó en alguna de las vigas trianguladas menores, de diseño más convencional (cordones a base de UPN apareados; montantes y diagonales a base de UPN o angulares apareados; tirantes formados por dos angulares en cruz o por dos chapas). La segunda variante (cartela adosada inferiormente) es de ejecución muy cómoda, pero tiene el peligro de poder coincidir con una zona de segregaciones no detectadas en los ensayos de recepción o condiciones equivalentes de garantía de calidad del material; no obstante, puesto que la amplitud de tales zonas de impurezas tiene que ser considerable para afectar a la capacidad última de la unión, y aun así queda siempre una capacidad residual variable, raramente inferior a $2 / 3$ del valor nominal incluso para material muy defectuoso, en

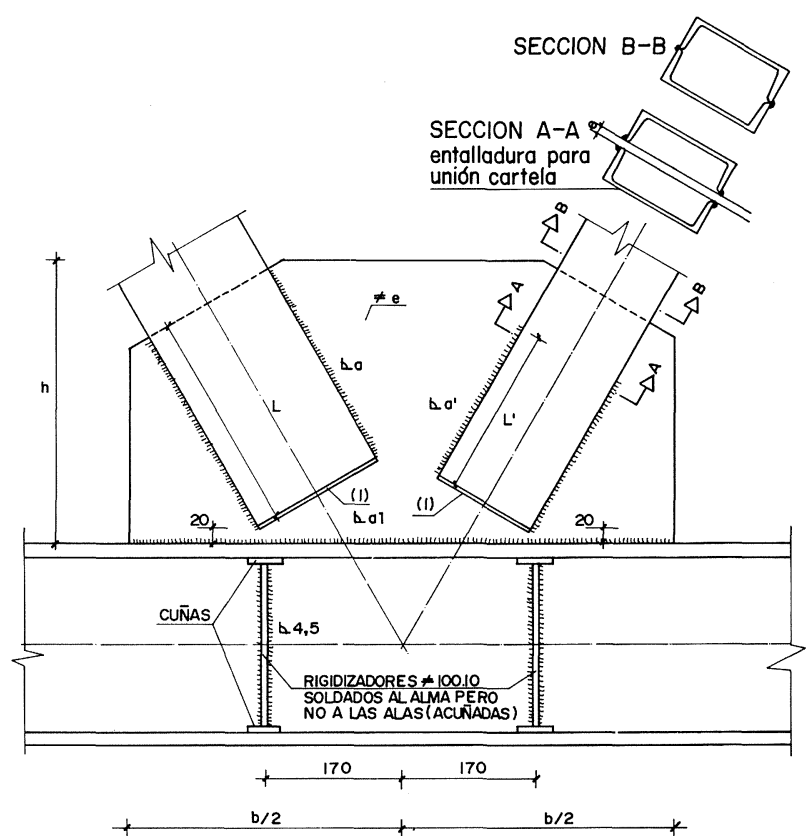

Fig. 4. - Nudo tipo, cordón inferior (cerchas principales).

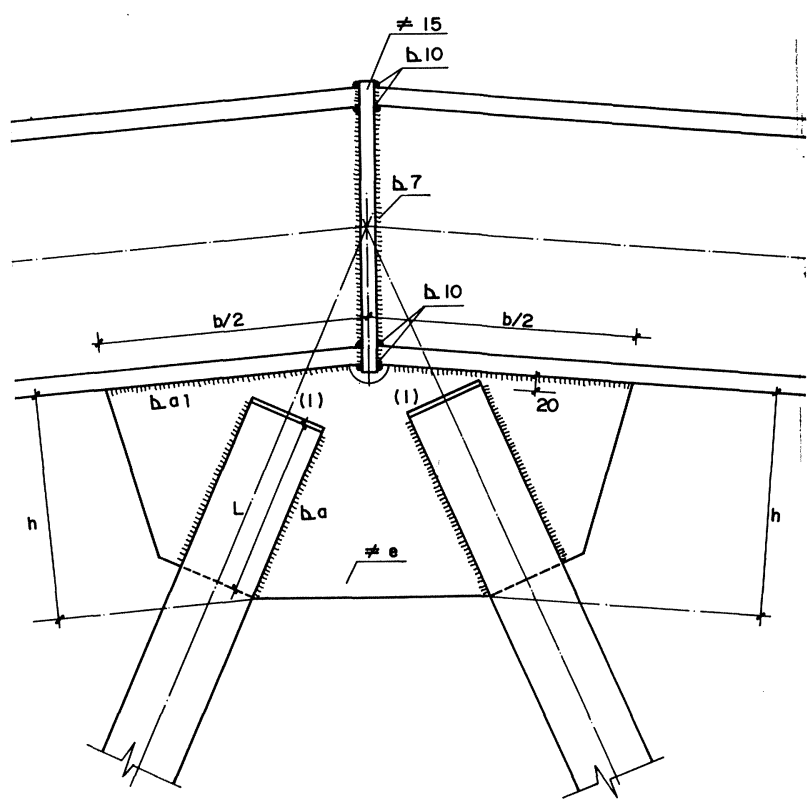

Fig. 5. - Nudo central, cordón superior (cerchas principales). 


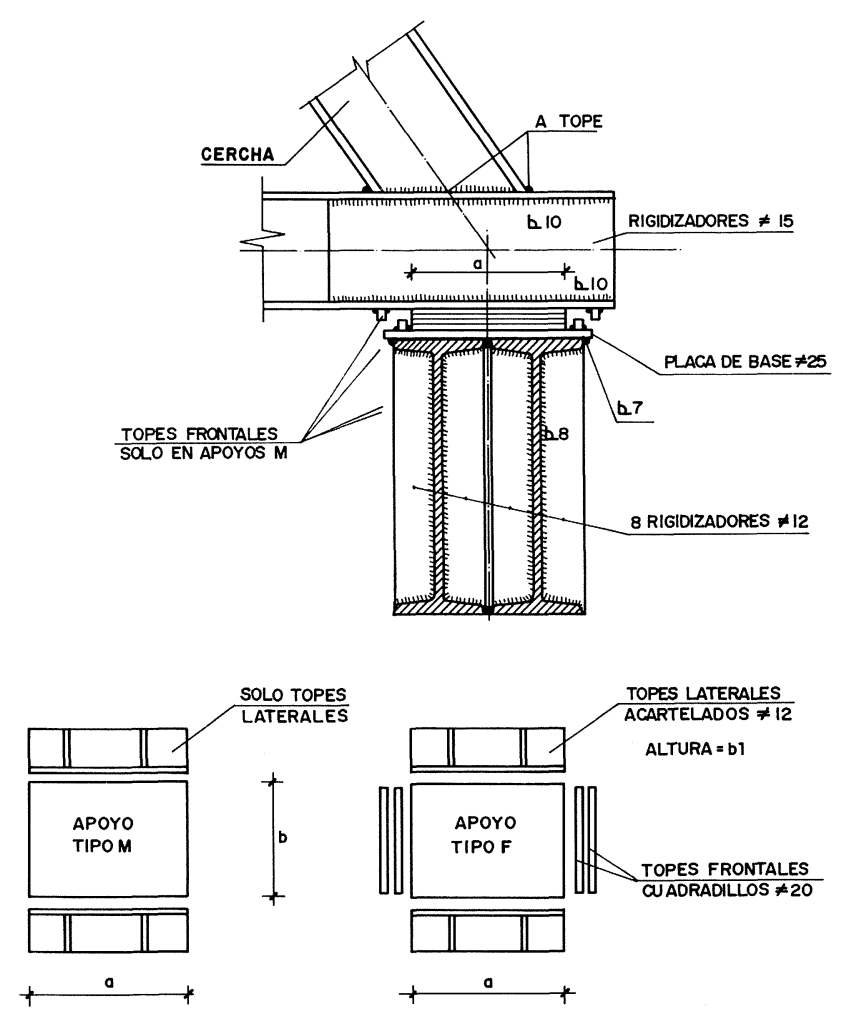

Fig. 6. - Apoyos de las cerchas principales sobre el dintel de borde.

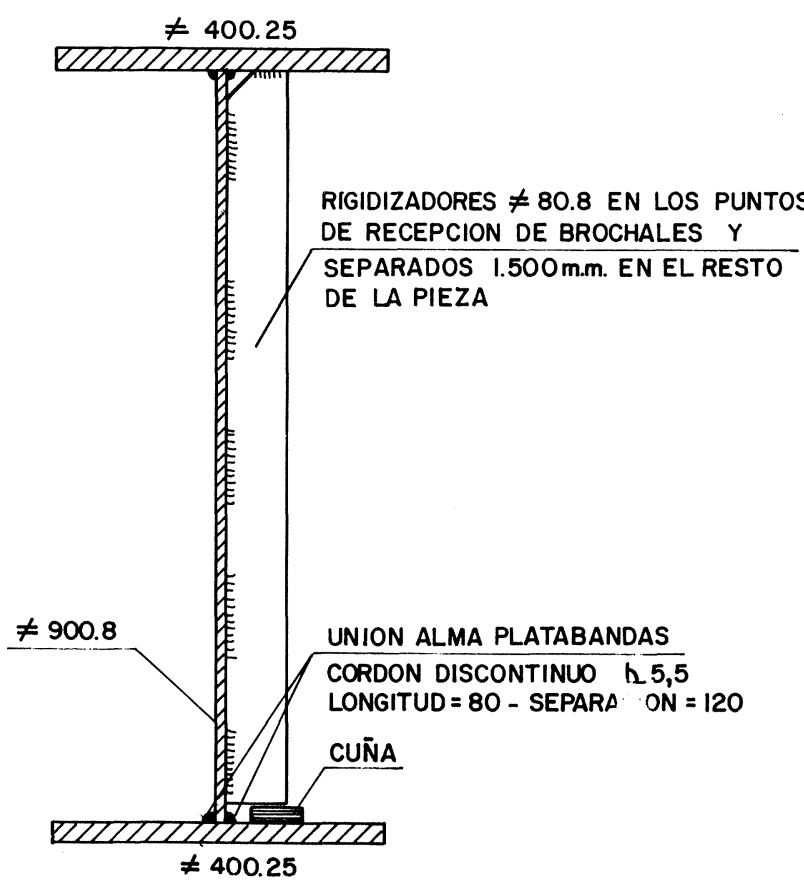

Fig. 7.-Viga arrraua vı.

caso de acero ordinario $\left({ }^{*}\right)$, se llegó a utilizar dicha variante en algún tirante poco solicitado suspendido de viga (no de cercha) tomando las precauciones de adoptar una cartela extensa y sobredimensionada, y de exigir el control por ensayos no destructivos

(*) V. al respecto los resultados experimentales publicados por B.D. MacDonald Journal of the Structural Div., ASCE, Aug. 1979, pp 1605-1619). Para aceros análogos al A52 español, la relación anterior de 2/3 /lega a reducirse claramente (1/2 y aun inferior).

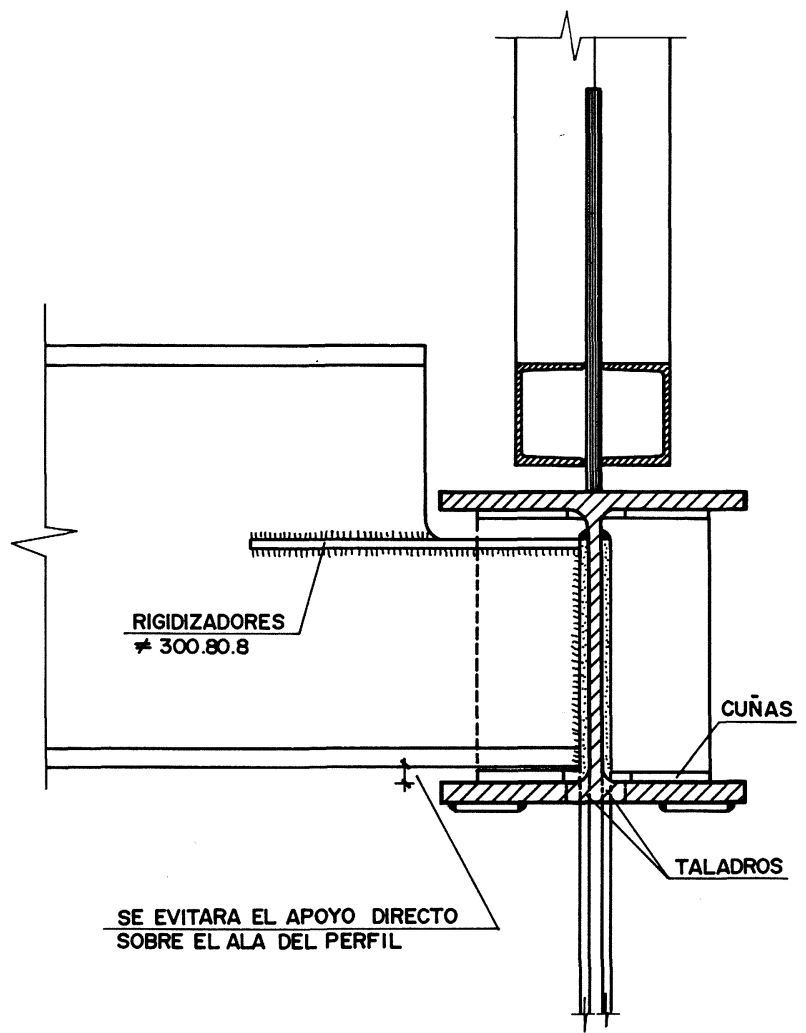

Fig. 8. - Unión de tirante y viga transversal.

(ultrasonidos o similar) específicamente de la zona de la viga afectada por la cartela, antes de la selección del escaso número de perfiles destinados a tal fin.

Para los tirantes principales se adoptó en todos los casos la solución de soldarlos directamente a ambos lados del alma del cordón inferior de las grandes piezas trianguladas, introduciendo el tirante por dos taladros alargados realizados en las alas inferiores de dicho cordón (el cual se reforzaba localmente para restituir la débil pérdida de sección ocasionada por esos taladros). La sección del tirante, constituida por dos chapas rectangulares, al ser doble y con sendas uniones independientes, asegura una mayor fiabilidad que la de una pieza simple. En la figura 8 se muestra el esquema del nudo, representando también la unión de una viga transversal que debía necesariamente recibirse a la misma cota en algunos nudos. De los comentarios efectuados por los responsables del montaje de la estructura metálica se infiere que la ejecución de estos nudos, y en general la de toda la obra, no planteó especiales dificultades de realización.

En la parte inferior de los tirantes se ha seguido el mismo criterio de unir directamente el tirante a las almas de las piezas, desmembrando las cabezas superiores de éstas (adecuadamente restituidas mediante platabandas, en caso de haber continuidad), lo que no impedía ejecutar con facilidad el apoyo simple de vigas transversales que coincidian con algunos tirantes (ver figuras 9 y 10). 


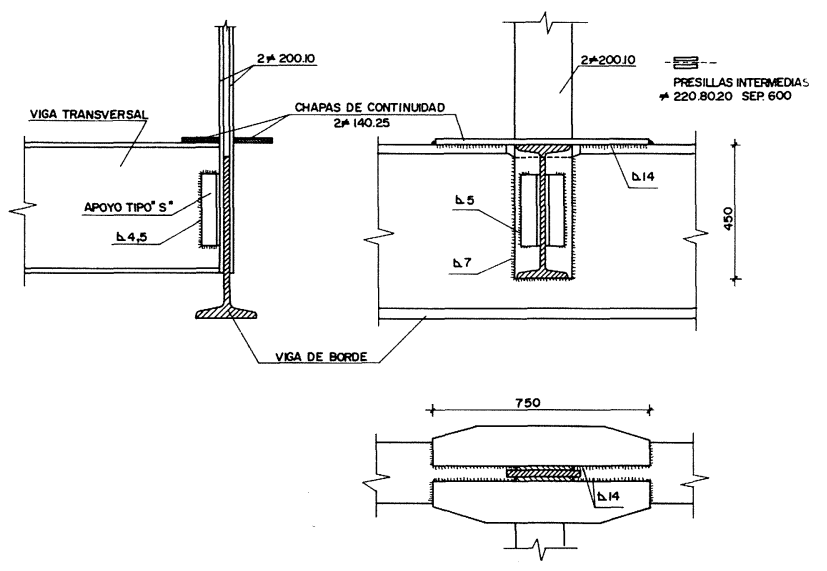

Fig. 9. - Tirantes $\mathrm{T} 3,4$ y 5 .

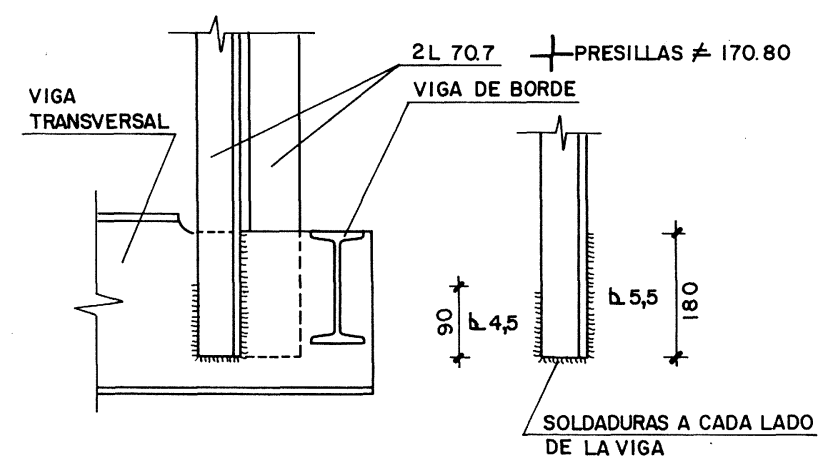

Fig. 10. - Tirantes T27, 28, 32, 39 y 47.

\section{Ejecución}

A instancias de la Dirección de obra se incluyó en el proyecto un Pliego de Condiciones, en el que se incluía un conjunto de directrices que pudieran servir de orientación para los responsables del control de calidad de la estructura metálica. Aparte de las pertinentes condiciones relativas al suministro y recepción de materiales, ejecución en taller $y$ en obra, tolerancias geométricas, calificación de soldadores, etc., se detallaron algunas condiciones más específicas, tales como el control por ensayos no destructivos de algunas zonas de perfiles que recibían tirantes (aspecto comentado en el apartado anterior) y un estudio relativo a los puntos de suspensión de las vigas principales durante el izado hasta su posición definitiva (proceso durante el cual convenía estudiar las condiciones de inestabilidad de grandes longitudes de barras aún no arriostradas). Pero la mayor atención se dedicó a establecer un conjunto de recomendaciones para el control de uniones soldadas, previéndose la inspección visual y control geométrico en casos ordinarios, y recomendándose la inspección radiográfica de las uniones de mayor responsabilidad.

\section{Equipo técnico}

- Estructura metálica: Jesús Ortiz Herrera, Juan Antonio Domínguez Montero y María Jesús Izquierdo Martínez, Ingenieros de Caminos y José Espinosa González, Aparejador.

- Estructura de hormigón armado: Carlos Siegrist Fernández y Manuel Martínez Lafuente, Dres. Ingenieros de Caminos.

- Dirección y coordinación del proyecto de estructura: Javier Manterola Armisén, Dr. Ingeniero de Caminos.

- Dirección de la obra: Manuel Blanc Díaz y Eduardo Marquina, Dres. Arquitectos y Heriberto Rueda Núñez, Santiago Sancho Ruiz y Javier Velasco Estévez, Aparejadores.

- Empresa Constructora: Construcciones Bigar.

- Control de uniones soldadas: Intemac.

\section{publicación del i.e.t.c.c.}

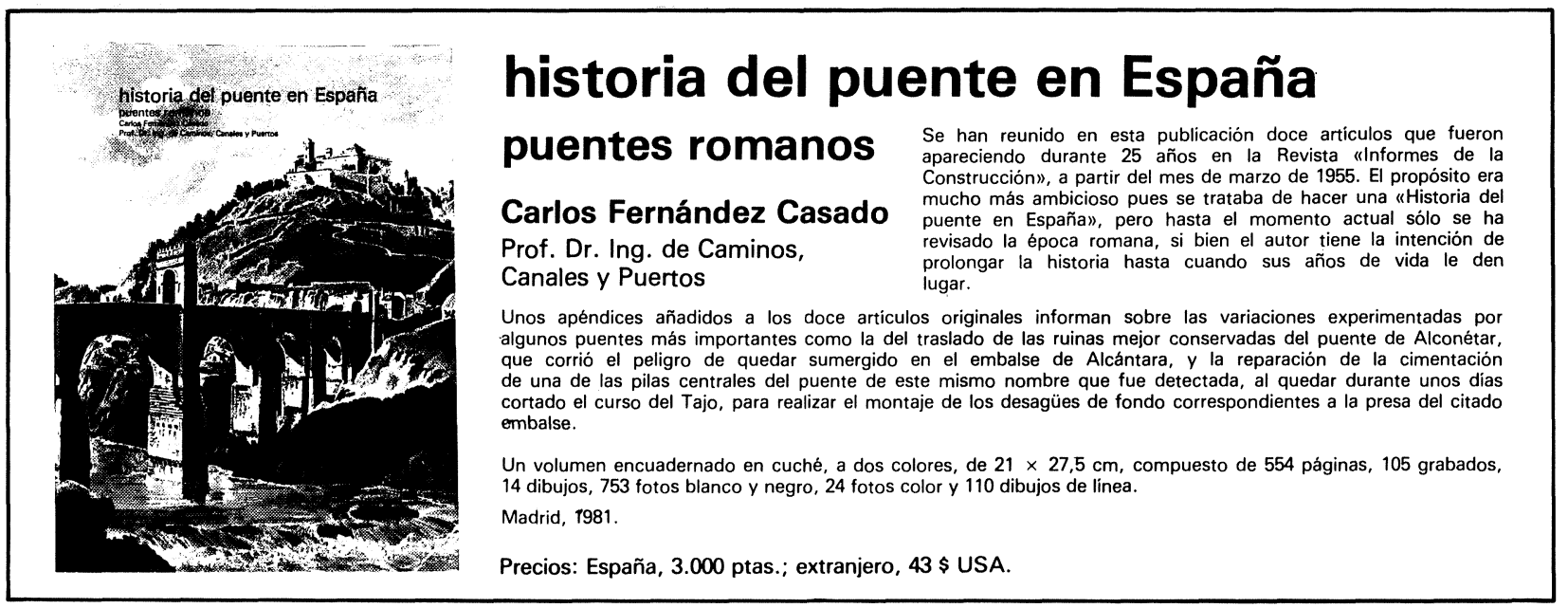

